\title{
COMPETING RISKS AFTER CORONARY BYPASS SURGERY: THE INFLUENCE OF DEATH ON REINTERVENTION
}

Eugene H. Blackstone, MD ${ }^{\mathrm{a}, \mathrm{b}}$

Bruce W. Lytle, $\mathrm{MD}^{\mathrm{b}}$
Objective: For groups of patients at high risk of death, such as older patients, the actual probability of experiencing a nonfatal event, such as reintervention, must be far smaller than the potential probability were there no attrition by death. Competing risks analysis quantifies the difference.

Methods: Multivariable analyses were performed for the competing events death before reintervention, reoperation, and percutaneous transluminal coronary angioplasty in 2001 patients after bilateral internal thoracic artery grafting and in 8123 after single internal thoracic artery grafting. Follow-up was $9.7 \pm 3.0$ years and $10.8 \pm 5.2$ years in bilateral and single internal thoracic artery groups, respectively.

Results: Patients receiving single grafts experienced shorter survival and more reinterventions $(P<.0001)$. However, other risk factors for death included old age $(P<.0001)$, but risk factors for reintervention included young age $(P<.0001)$. This difference confounds interpretation of eventfree survival that is clarified by competing risks analysis. Death reduced the potential benefit of bilateral internal thoracic artery grafting on reintervention by angioplasty from a median of $8.5 \%$ to $5.5 \%$ at 12 years and by reoperation from $9.3 \%$ to $6.8 \%$, with progressively greater erosion of benefit from attrition by death as age increased. Competing risks simulation confirmed that young age was a true risk factor for reintervention, excluding the explanation that it reflected simply passive attrition by death as patients age.

Conclusions: Even after accounting for attrition by interim deaths, bilateral versus single internal thoracic artery grafting and older age are associated with fewer reinterventions. However, in high-risk patients, its benefit on freedom from reintervention is eroded considerably by death. ( $\mathrm{J}$ Thorac Cardiovasc Surg 2000;119:1221-32)
$\mathrm{H}$ igh-risk patients, like the elderly, may not survive to reap the long-term benefit of fewer reinterventions after receiving two versus one internal thoracic artery (ITA) grafts. ${ }^{1}$ Are the benefits of bilateral ITA grafting on reintervention nullified in high-risk patients?

From the Department of Thoracic and Cardiovascular Surgery and the Department of Biostatistics and Epidemiology, ${ }^{\text {b }}$ The Cleveland Clinic Foundation, Cleveland, Ohio.

Read at the Seventy-ninth Annual Meeting of The American Association for Thoracic Surgery, New Orleans, La, April 18-21, 1999.

Received for publication April 22, 1999; revisions requested Aug 31, 1999; revisions received Jan 26, 2000; accepted for publication Feb 16, 2000.

Address for reprints: Eugene H. Blackstone, MD, Department of Thoracic and Cardiovascular Surgery, 9500 Euclid Ave, Desk F25, Cleveland, OH 44195 (E-mail: blackse@ ccf.org).

Copyright () 2000 by The American Association for Thoracic Surgery

$0022-5223 / 2000 \$ 12.00+0 \quad \mathbf{1 2 / 6 / 1 0 6 5 1 9}$

doi: $10.1067 / \mathrm{mtc} .2000 .106519$
Reinterventions after bilateral ITA grafting are fewer in the elderly. ${ }^{1-3}$ Does the decreased incidence of reintervention in the elderly simply reflect passive attrition by death?

The benefits of different interventions, such as bilateral versus single ITA grafting, are commonly expressed in terms of event-free survival. However, because reintervention is prominent in the young and death in the elderly, a young patient and an old patient may have similar event-free survival estimates. Can event-free survival be presented in a less counterintuitive format?

To answer these questions, we need to adjust potential long-term benefits for attrition by death. Competing risks analysis can accomplish this. ${ }^{4,5}$ Therefore, we reexamined the outcome of patients after elective primary isolated coronary revascularization using one or two ITA grafts in light of three competing time-related events-death, reoperation, and percutaneous translu- 
minal coronary angioplasty (PTCA)—which together constitute event-free survival.

\section{Patients and methods}

Patients. Elective primary isolated bilateral ITA operations were performed in 2001 patients at The Cleveland Clinic Foundation between 1971 and January 1990. During this time frame, 8123 patients received single ITA grafting and one or more venous grafts among the first 1000 patients each year undergoing coronary revascularization. Both groups have been characterized. ${ }^{1}$

Patients receiving bilateral ITA grafts differed in many respects from those receiving single ITA grafts. ${ }^{1}$ Therefore, nonparsimonious propensity matching on known variables was used to formulate a well-matched, comparable group of 1975 patients undergoing bilateral ITA grafting and 4147 undergoing single ITA grafting. ${ }^{1}$ These patients were the basis for the competing risks analyses in the present study.

Event-free survival and competing risks. Construction of an event-free survival curve and conduct of a competing risks analysis begin identically. The earliest occurrence of one of three mutually exclusive events was considered: (1) death, (2) cardiac reoperation, and (3) PTCA. The common analysis interval was the interval between operation and the earliest occurrence of one of these events. Freedom from all events (alive without reintervention) is termed event-free survival.

Freedom from each event was estimated by the nonparametric product-limit method (formula 4.4.2 of Andersen and associates ${ }^{6}$ ). Variances of the estimates were based on the Greenwood formula (formula 4.4.19 of Andersen and associ$\left.a^{a t e s}{ }^{6}\right)$. Asymmetric confidence limits were calculated with the use of these variances and formula 6E-6 in Kirklin and Barratt-Boyes. ${ }^{7}$ The instantaneous risk (hazard function) for each competing event was estimated by a parametric method* that resolved the number of hazard phases, identified the shape of the hazard function, and estimated its parameters. ${ }^{8}$ The width of the confidence limits for estimates calculated from the resulting equations were consistent in width with those for nonparametric estimates.

Multivariable analyses. Variables examined multivariably for each event were listed previously. ${ }^{1}$ Multivariable analyses were conducted independently in the multiphase hazard function domain ${ }^{8}$ for each of the three competing events to generate parsimonious equations. Regression coefficients are presented plus or minus one standard error. These are presented rather than hazard ratios because the models, and the underlying data, were not proportional hazards across time.

Because the mode of reintervention changed across calendar time, multivariable logistic analysis was performed of reintervention by PTCA versus reoperation (Appendix I).

Synthesis of information. Consequences of the independent, simultaneously operative, migration rates (hazard functions) from the category alive without reintervention (eventfree survival) into each of the event categories were obtained by integration with the use of the parametric equations (Appendix II). This was supplemented, in some instances,

*Available by anonymous ftp://uabcvsr.cvsr.uab.edu for the SAS system. with a comparison of the corresponding probability of the reintervention, obtained from the same multivariable equations, with its competing risks counterpart. The familiar probability of reintervention applies to patients who are alive and have not yet experienced the event. It represents their potential risk of reintervention were the competing risk of death not present. Thus, two different presentations are possible from the identical hazard functions: potential, from reintervention probability estimates, and actual, from the percent of patients expected to experience reintervention in the context of multiple hazard functions operating simultaneously., ${ }^{9}, 10$

These analyses were the substrate used to address the questions posed in the introduction. The question sequence will be altered to more easily describe the methods used for answering them.

Can event-free survival be presented in a less counterintuitive format? Event-free survival is a composite time-related entity representing the aggregate occurrences of several dissimilar events. In this study, event-free survival decreases across time, commensurate with the percent of patients who have died, undergone reoperation, or experienced a PTCA increase across time. From one type of patient to another, and across time, the magnitude of event-free survival may be dominated by a different event. We propose that the simultaneous display of all components of event-free survival clarifies which events dominate and when, permitting clear interpretation of what may be otherwise counterintuitive aspects of event-free survival.

Are the benefits of bilateral ITA grafting on reintervention nullified in high-risk patients? Both potential and actual expected benefits of two versus one ITA graft were compared for each patient. To accomplish this, we solved the multivariable equations for each patient (1) as if single ITA grafting had been performed and (2) again as if bilateral ITA grafting had been performed. For these calculations, the date of operation was artificially set to January 1, 1990, because of the prominent influence of calendar date on type of outcome (Appendix I). The difference between having one or two ITA grafts in the probability of each event (potential) and again in the proportion of events (actual) is presented at 12 years postoperatively.

Does the decreased incidence of reintervention in the elderly simply reflect passive attrition by death? To investigate this question, we performed a competing risks simulation. Multivariable equations for reoperation and for PTCA were generated exactly as will be presented in Tables II and III, except the age association was ignored. Three independent uniform random number generators were used to generate a separate probability of death, reoperation, and PTCA for each simulated patient. The interval from operation to the time of each generated probability was calculated from the inverse of the multivariable equations. If the simulated interval to an event exceeded 12 years, the time was censored. A common interval to the earliest of simulated death, reoperation, or PTCA was generated. Finally, multivariable analyses of reoperation and PTCA were performed on these artificial events and intervals (with actual patient values being used for all risk factors) to determine if the coefficients for age in the reintervention analyses were different from the anticipated 

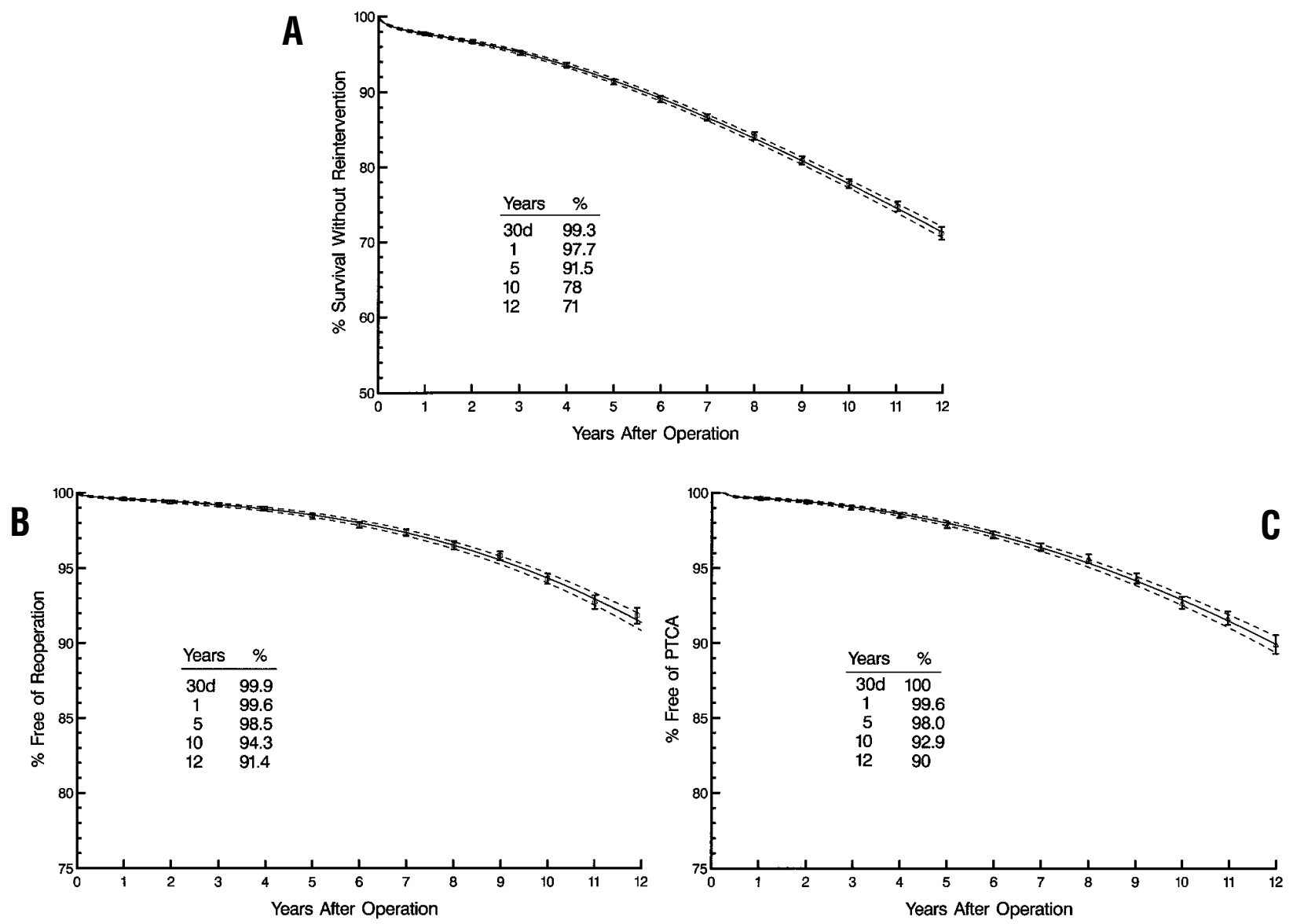

Fig 1. Overall freedom from three mutually exclusive competing risks after single or double ITA grafting. Because of the density of information, product-limit nonparametric estimates are shown only at yearly intervals accompanied by asymmetric $68 \%$ confidence limits (equivalent to one standard error), depicted by the vertical bars. Parametric estimates are shown as continuous solid lines enclosed within dashed $68 \%$ confidence limits. The number of patients remaining at risk at 2, 4, 6, 8, 10, and 12 years was 5747, 5484, 5433, 3807, 2953, and 658. A, Survival without reintervention. Notice the expanded vertical axis and close correspondence between nonparametric (symbols) and parametric estimates. B, Freedom from reintervention by reoperation. Notice the greatly expanded vertical axis. $\mathbf{C}$, Freedom from reintervention by PTCA. Note the expanded vertical scale.

value of zero. A negative coefficient would be evidence that the risk factor analyses of reintervention were influenced spuriously by passive attrition from death of old patients.

\section{Results}

Overall risk-unadjusted competing risks. The bases for the competing risks analysis were the mutually exclusive time-related events depicted in Fig 1: death before reintervention, reoperation, and PTCA. These depictions demonstrate a close correspondence between nonparametric and parametric methods of estimation. The competing risks calculations were driven by the rates of migration of patients from the initial category of being alive without reintervention (eventfree survival) into one of the three categories of events (Fig 2). The cumulative result of the three migration rates acting simultaneously across time is shown in Fig 3. Event-free survival diminished as the percent of patients experiencing either death before reintervention or one of the two modes of reintervention accumulated. At every point in time, the percent of patients in each of the four categories added to $100 \%$.

Although the hazard functions generating Figs 1 and 3 were identical, Fig 1 depicts the pure probability of events among living patients, whereas Fig 3 depicts the end result of accounting for events simultaneously as they remove patients from risk. Thus, at 12 years the probability of each event among living patients (Fig 1) versus the percent of patients that will experience the event before experiencing any other (Fig 3) were $28.7 \%$ versus $26.6 \%$ for death, $8.6 \%$ versus $6.8 \%$ for reoperation, and $10.1 \%$ versus $8.1 \%$ for PTCA. 


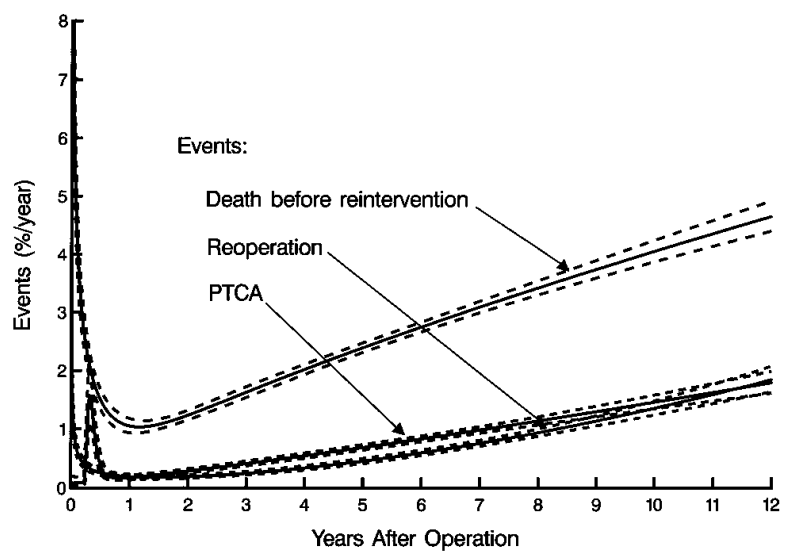

Fig 2. Risk-unadjusted migration rates (hazard functions) into each of the three mutually exclusive event categories: death before reintervention, reintervention by reoperation, and reintervention by PTCA. The figure's format is similar to that of Fig 1.

Multivariable analyses. The overall competing risks analysis has little intrinsic value because the transition rates are modulated importantly by patient variables (risk factors). The risk factors were summarized qualitatively and discussed previously ${ }^{1}$ but are presented quantitatively in Tables I through III.

Single ITA grafting was associated with a greater probability of death, reoperation, and PTCA than was bilateral ITA grafting. However, the risk factors for death, in contrast to those for reoperation or PTCA, included a strong relation to old rather than young age at operation, to greater left ventricular dysfunction than less, and to several comorbidities rather than none. It was the opposing direction of influence of risk factors and the presence of different risk factors for different events that made estimates of event-free survival counterintuitive.

Can event-free survival be presented in a less counterintuitive format? Yes.

Fig 4 contrasts event-free survival and the individual competing risk components between bilateral and single ITA grafting in a quite young (age 35 years), but otherwise median-risk, patient. Death was unlikely, regardless of surgical strategy. Thus, the dominant event determining event-free survival was reintervention.

Similar event-free survival and competing risks component curves were constructed across a wide spectrum of ages and compared at 12 years (Fig 5). Event-free survival at 12 years was arching, meaning that after single ITA grafting (Fig 5, B) event-free survival was identical for a 30-year-old patient and a 66-year-old patient.

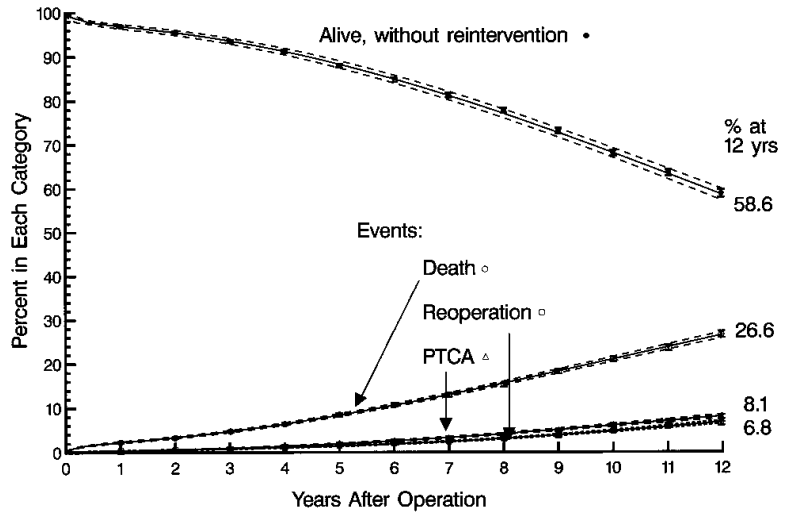

Fig 3. Competing risks depiction of the consequences of the three migration rates shown in Fig 2. These rates deplete the proportion $(\%)$ of patients alive and without reintervention (filled circles) and increase the proportion dying before reintervention (circles) or experiencing reintervention by reoperation (squares) or PTCA (triangles). At all points in time, the percentage of patients in all categories sum to $100 \%$, as shown in the percents at 12 years in the right margin of the figure.

The reason for this counterintuitive finding is clarified by the competing risks display of the individual components constituting event-free survival: the 30year-old patient's event-free survival estimate was dominated by low-risk PTCA and the 66-year-old patient's by death.

Are the benefits of bilateral ITA grafting on reintervention nullified in high-risk patients? No.

Across all ages, fewer patients were predicted to undergo reintervention with bilateral rather than single ITA grafting. ${ }^{1}$ However, at old age, death prominently reduced the percent of patients who potentially might have required reintervention. Therefore, the potential probability of reintervention among surviving older patients was higher than the actual percent of older patients expected to experience a reintervention because many died before that point (Fig 6). This progressively narrowed the predicted benefit of two versus one ITA graft as age increased.

The difference between estimates of potential probability of reintervention on the one hand, and actual percent of patients expected to experience one on the other, increased dramatically for patients at highest risk of death. For example, a diabetic 70-year-old man with three-system disease and left ventricular dysfunction had a predicted $16 \%$ probability of reoperation and $9 \%$ probability of PTCA if he survived 12 years, but only a $1.5 \%$ chance of actually experiencing a reoperation and 
Table I. Risk factors for death before reintervention after single or bilateral ITA grafting in propensity-matched patients

\begin{tabular}{|c|c|c|c|}
\hline Incremental risk factor & Coefficient $\pm S D$ & Direction of influence & $\mathrm{P}$ \\
\hline \multicolumn{4}{|l|}{ Early hazard phase } \\
\hline \multicolumn{4}{|l|}{ Demography } \\
\hline Women & $0.65 \pm 0.21$ & $\uparrow$ & .002 \\
\hline Age $^{*}$ & $0.99 \pm 0.181$ & $\uparrow$ & $<.0001$ \\
\hline \multicolumn{4}{|l|}{ Left ventricular function } \\
\hline LV dysfunction ${ }^{\dagger}$ & $0.25 \pm 0.052$ & $\uparrow$ & $<.0001$ \\
\hline \multicolumn{4}{|l|}{ Comorbidity } \\
\hline Peripheral vascular disease & $1.02 \pm 0.22$ & $\uparrow$ & $<.0001$ \\
\hline \multicolumn{4}{|l|}{ Operation } \\
\hline ITA to $\mathrm{LAD}^{\ddagger}$ & $-0.57 \pm 0.27$ & $\downarrow$ & .03 \\
\hline Date of operation ${ }^{\S}$ & $1.92 \pm 0.74$ & $\downarrow$ & .01 \\
\hline \multicolumn{4}{|l|}{ Late hazard phase } \\
\hline \multicolumn{4}{|l|}{ Demography } \\
\hline Age & $0.094 \pm 0.0115$ & $\uparrow$ & $<.0001$ \\
\hline Age & $0.71 \pm 0.28$ & $\downarrow$ & .01 \\
\hline \multicolumn{4}{|l|}{ Symptoms } \\
\hline Angina severity & $0.072 \pm 0.026$ & $\uparrow$ & .006 \\
\hline \multicolumn{4}{|l|}{ Coronary disease } \\
\hline Left main trunk disease ${ }^{\mathbb{I}}$ & $0.26 \pm 0.122$ & $\uparrow$ & .03 \\
\hline No. of diseased coronary systems & $0.20 \pm 0.069$ & $\uparrow$ & .003 \\
\hline \multicolumn{4}{|l|}{ Left ventricular function } \\
\hline LV dysfunction & $0.67 \pm 0.184$ & $\uparrow$ & .0003 \\
\hline And age & $-0.39 \pm 0.149$ & $\downarrow$ & .009 \\
\hline History of MI & $0.21 \pm 0.073$ & $\uparrow$ & .004 \\
\hline History of CHF & $0.74 \pm 0.116$ & $\uparrow$ & $<.0001$ \\
\hline \multicolumn{4}{|l|}{ Comorbidity } \\
\hline Pharmacologically treated diabetes & $2.5 \pm 1.21$ & $\uparrow$ & .04 \\
\hline And age & $-4.7 \pm 2.4$ & $\uparrow$ & .05 \\
\hline And age $e^{* *}$ & $3.0 \pm 1.11$ & $\downarrow$ & .006 \\
\hline Hypertension & $0.35 \pm 0.069$ & $\uparrow$ & $<.0001$ \\
\hline Smoking history & $0.44 \pm 0.068$ & $\uparrow$ & $<.0001$ \\
\hline PVD & $0.44 \pm 0.082$ & $\uparrow$ & $<.0001$ \\
\hline \multicolumn{4}{|l|}{ Operation } \\
\hline Single ITA grafting & $0.28 \pm 0.070$ & $\uparrow$ & $<.0001$ \\
\hline
\end{tabular}

$I T A$, Internal thoracic artery; $S D$, standard deviation; $L V$, left ventricular; $L A D$, left anterior descending coronary artery; $M I$, myocardial infarct; $C H F$, chronic heart failure; $P V D$, peripheral vascular disease.

*exp[age/50] natural exponential function.

'[LV dysfunction grade $]^{2}$ squared transformation.

Negative sign of coefficient indicates a lesser probability of death before reintervention.

$\S[1 /$ number of years since January 1,1971$]$ inverse transformation.

$\|[50 / \text { age }]^{2}$ squared inverse transformation.

I[ [\% stenosis/100]scaled transformation.

\#[50/age] inverse transformation; interaction with diabetes.

**[50/age $]^{2}$ squared inverse transformation; interaction with diabetes.

$1 \%$ chance of actually experiencing a PTCA because of the $97.5 \%$ chance of dying in the interim.

These considerations permit us to summarize not just the potential benefit of bilateral ITA versus single ITA grafting, but the actual benefit in terms of fewer reinterventions before death (Fig 7). The actual benefit was positive, but uniformly smaller than that expected on the basis of differences in probability because the attrition of patients by death increasingly dominated nonfatal events as age increased.

\begin{abstract}
Does the decreased incidence of reintervention in the elderly simply reflect passive attrition by death? No.

Simulated competing risks analyses that assumed no age effect related to reintervention still demonstrated that fewer older patients would survive to experience either reoperation or PTCA. However, multivariable risk factor analysis of simulated reintervention did not identify age as a risk factor and, more specifically, did not identify it as a negative risk factor. It yielded a coef-
\end{abstract}


Table II. Risk factors for reoperation after single or bilateral ITA grafting in propensity-matched patients

\begin{tabular}{|c|c|c|c|}
\hline Incremental risk factor & Coefficient $\pm S D$ & Direction of influence & $\mathrm{P}$ \\
\hline \multicolumn{4}{|l|}{ Early hazard phase } \\
\hline \multicolumn{4}{|l|}{ Comorbidity } \\
\hline Hypertension & $0.96 \pm 0.42$ & $\uparrow$ & .02 \\
\hline \multicolumn{4}{|l|}{ Operation } \\
\hline Single ITA grafting & $1.45 \pm 0.45$ & $\uparrow$ & .001 \\
\hline And hypertension & $3.3 \pm 0.77$ & $\uparrow$ & $<.0001$ \\
\hline Date of operation* & $-1.01 \pm 0.54$ & $\downarrow$ & .06 \\
\hline \multicolumn{4}{|l|}{ Late hazard phase } \\
\hline \multicolumn{4}{|l|}{ Demography } \\
\hline $\mathrm{Age}^{\dagger}$ & $-0.39 \pm 0.143$ & $\downarrow$ & .007 \\
\hline Height & $-0.023 \pm 0.0091$ & $\downarrow$ & .01 \\
\hline \multicolumn{4}{|l|}{ Left ventricular function } \\
\hline History of CHF & $0.75 \pm 0.31$ & $\uparrow$ & .02 \\
\hline \multicolumn{4}{|l|}{ Operation } \\
\hline Single ITA grafting & $1.79 \pm 0.30$ & $\uparrow$ & $<.0001$ \\
\hline ITA to $\mathrm{LAD}^{\ddagger}$ & $-0.52 \pm 0.198$ & $\downarrow$ & .008 \\
\hline ITA to RCA & $0.81 \pm 0.39$ & $\uparrow$ & .04 \\
\hline
\end{tabular}

$I T A$, Internal thoracic artery; $S D$, standard deviation; $C H F$, chronic heart failure; $L A D$, left anterior descending coronary artery; $R C A$, right coronary artery. * $\ln$ [number of years since January 1, 1971] natural logarithmic transformation.

exp[age/50] natural exponential transformation.

${ }^{\dagger}$ Negative sign of coefficient indicates a lesser probability of reoperation.

Table III. Risk factors for PTCA after single or bilateral ITA grafting in propensity-matched patients

\begin{tabular}{|c|c|c|c|}
\hline Incremental risk factor & Coefficient $\pm S D$ & Direction of influence & $\mathrm{P}$ \\
\hline \multicolumn{4}{|l|}{ Early hazard phase } \\
\hline None & - & & - \\
\hline \multicolumn{4}{|l|}{ Constant hazard phase } \\
\hline None & - & & - \\
\hline \multicolumn{4}{|l|}{ Late hazard phase } \\
\hline \multicolumn{4}{|l|}{ Demography } \\
\hline Age & $-0.522 \pm 0.0082$ & $\uparrow$ & $<.0001$ \\
\hline \multicolumn{4}{|l|}{ Coronary disease } \\
\hline RCA stenosis ${ }^{*}$ & $-0.52 \pm 0.168$ & $\downarrow$ & .002 \\
\hline \multicolumn{4}{|l|}{ LV function } \\
\hline LV dysfunction ${ }^{\dagger}$ & $-0.041 \pm 0.0121$ & $\downarrow$ & .0007 \\
\hline History of MI & $-1.76 \pm 0.66$ & $\downarrow$ & .008 \\
\hline And age & $0.032 \pm 0.0116$ & $\uparrow$ & .005 \\
\hline \multicolumn{4}{|l|}{ Operation } \\
\hline Single ITA grafting & $0.65 \pm 0.130$ & $\uparrow$ & $<.0001$ \\
\hline Date of operation ${ }^{\S}$ & $1.75 \pm 0.27$ & $\uparrow$ & $<.0001$ \\
\hline
\end{tabular}

$I T A$, Internal thoracic artery; $S D$, standard deviation; $R C A$, right coronary artery; $L V$, left ventricular; $M I$, myocardial infarct.

* $[\%$ stenosis/100] scaled transformation.

'[Grade of LV dysfunction] $]^{2}$ squared transformation.

Negative sign of coefficient indicates a lesser probability of PTCA.

$\S$ ln [number of years since January 1, 1971] natural logarithmic transformation.

ficient of nearly zero $(-0.0094$ in the early hazard phase and 0.0049 in the late hazard phase for reoperation and 0.00057 for PTCA). Thus, attrition by death, precluding reintervention in many patients, did not confound risk factor identification and quantification of independent nonfatal events.

\section{Discussion}

Principal findings. The combined use of competing risks analysis and simulation have achieved the three objectives of this study as embodied by three questions. Are the benefits of bilateral ITA grafting on reintervention nullified in high-risk patients? No. 

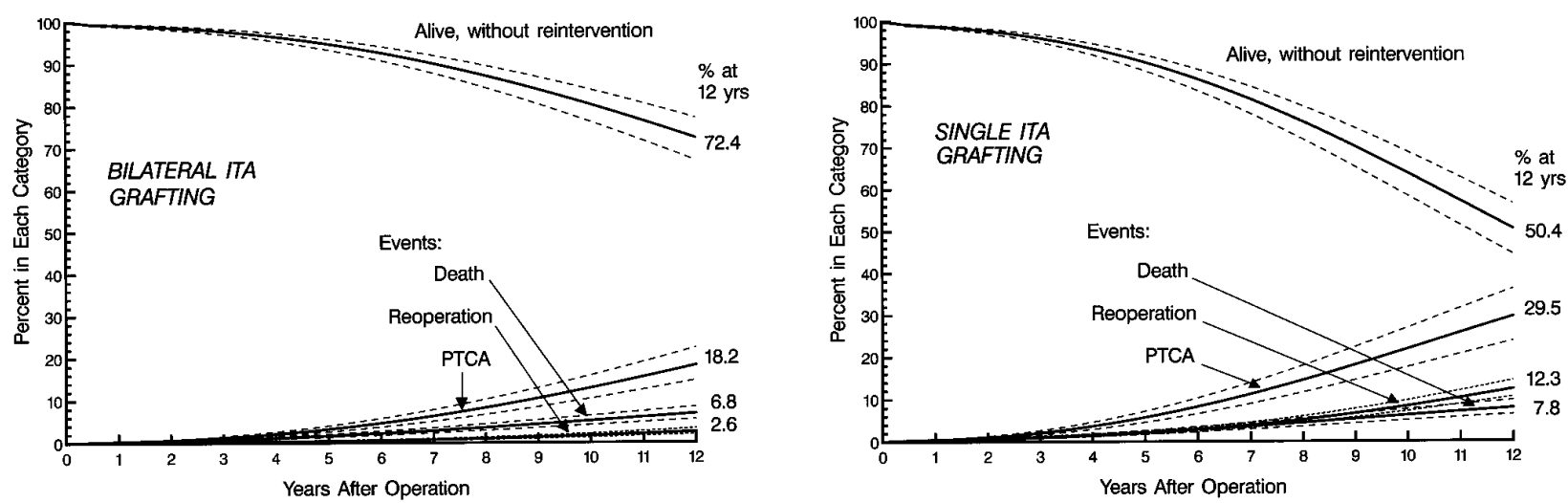

A

Fig 4. Competing risks presentation for a 35-year-old patient with median risk factors. The figure represents a solution to each of the parametric equations in Tables I to III, for which the following values for variables were provided: grade 3 angina, three-system disease, mild reduction of left ventricular function from a previous myocardial infarction, hypertension, no diabetes, no smoking, and an ITA graft to the left anterior descending coronary artery. The date of operation was set to January 1, 1990. The equations are predicting events over the subsequent 12 years. Point estimates are enclosed by dashed $68 \%$ confidence limits. A, Competing risks for bilateral ITA grafting. B, Competing risks for single ITA grafting.
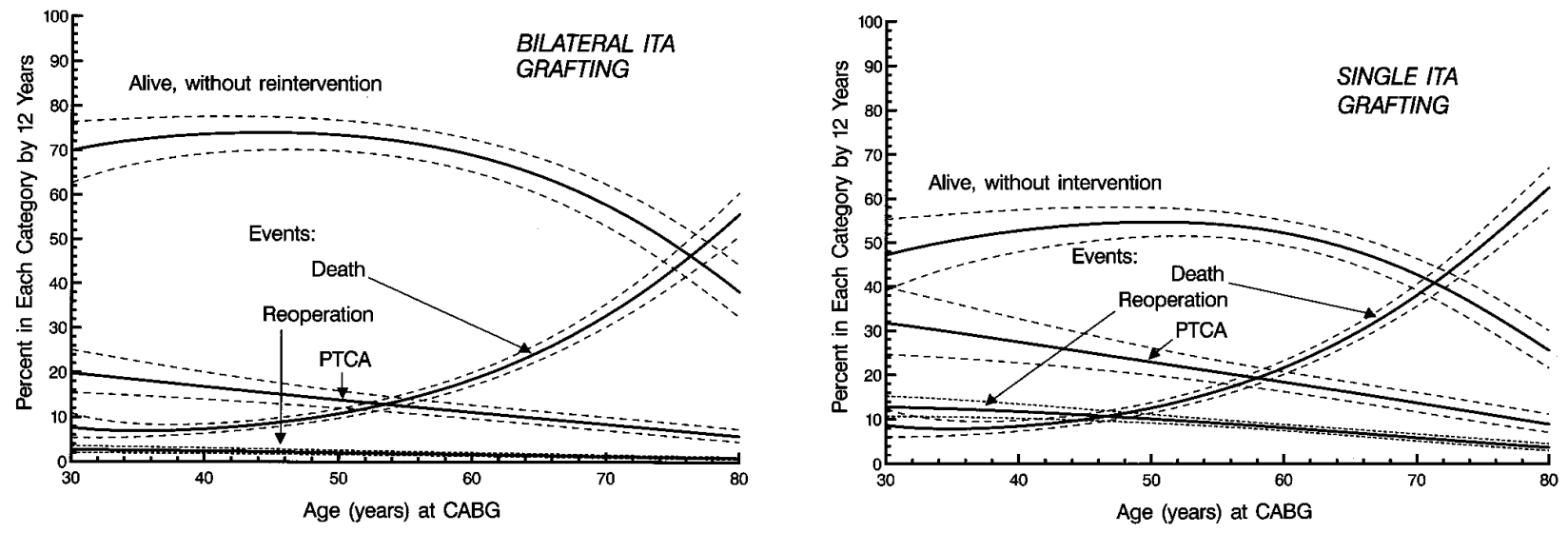

A

Fig 5. Proportion (\%) of patients in each event category by 12 years according to the age of the patient at CABG. Values used to solve the parametric equations were otherwise identical to those in Fig 4. Point estimates are enclosed by dashed $68 \%$ confidence limits. A, Competing risks for bilateral ITA grafting. B, Competing risks for single ITA grafting. Note the counterintuitive, but accurate, finding that the estimates of event-free survival (upper convex curve) are identical at both extremes of age.

The actual long-term benefit of bilateral versus single ITA grafting on freedom from reintervention was considerably less for some patients, such as the elderly, than those calculated from differences in reintervention probabilities. Thus, despite the fact that the actual number of reinterventions in the elderly will be fewer than the potential number were survival not the issue, bilateral ITA grafting still decreases the actual number of reinterventions in the elderly compared with single
ITA grafting. This observation of decreased realized long-term benefit by factors that importantly reduce the likelihood of long-term survival was emphasized by Sergeant, Blackstone, and Meyns ${ }^{12}$ for vein grafts versus use of the ITA.

Rates of migration from event-free survival to death, reoperation, or PTCA were influenced by both patientrelated and operation-related variables; however, age was the most important patient-related variable and the 


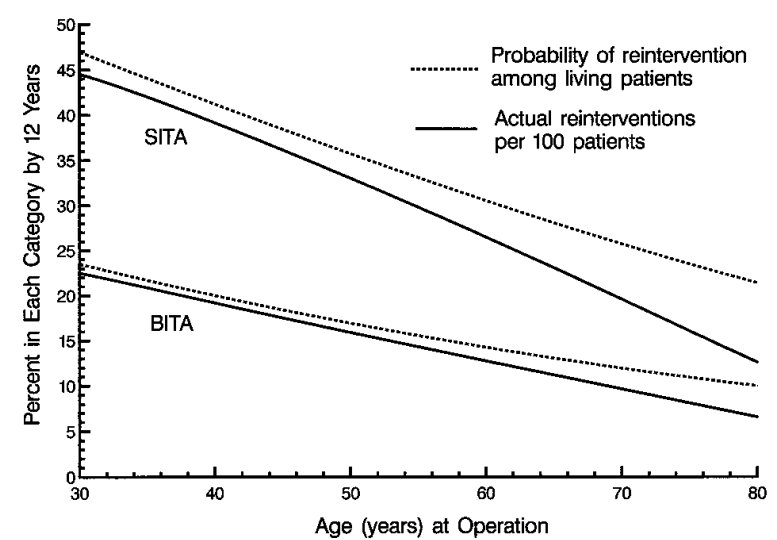

Fig 6. Prevalence of reoperation predicted from the competing risks analysis (solid lines) and estimated from the probability of reintervention among living patients (dashed lines). The characteristics of the patients are as in Figs 4 and 5. Neither death nor patients alive without reintervention are depicted on this figure for clarity. The solid and dashed lines are derived from the same risk factor equation (Table II); however, the solid line is adjusted for occurrence of interim deaths and reinterventions by PTCA.

easiest to portray. It was not the only one. Thus, it is important to assess the influence of multiple patientrelated variables that determine the difference between the potential and the actual benefit of different surgical strategies for both individuals and groups of patients.

The inevitable question arises as to whether one should concentrate on individual event estimates, as is true of conventional analyses, or on competing risks estimates that are emphasized in this study. We believe both have a complementary and different role in patient-physician decision making, generation of new knowledge, and understanding of heart disease and its treatment. Conventional individual event analyses isolate the effects of single end points, removing the confounding influence of other events so as to reveal the nature of the disease or treatment on a specific event. In contrast, the competing risks analysis uses this same information, the same hazard functions, and the same risk factor analyses to reveal the consequences of these events operating simultaneously.

Does the decreased incidence of reintervention in the elderly simply reflect passive attrition by death? No.

The depletion by death of patients at risk of reintervention does not result in spurious risk factor analyses and interpretation so long as the competing events are reasonably independent. Thus, young age is a true risk factor for reintervention, not the spurious, passive result of attrition by death of older patients. This find-

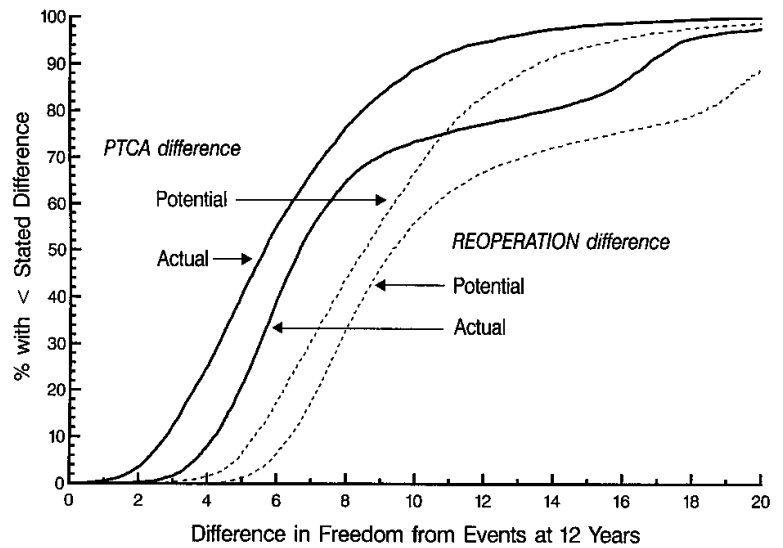

Fig 7. Cumulative frequency distribution of the difference in freedom from reintervention at 12 years were each patient to receive a bilateral versus a single ITA grafting operation. The solid lines are the differences in events estimated to actually occur, given the possibility of interim events. The corresponding lightly dashed lines are the differences based on individual probabilities of the events (potential benefits were patients to remain alive). The difference between actual and potential benefit is due to attrition by death. [Note: The "potential benefits" are numerically different from those in our previous article ${ }^{1}$ because for this depiction we artificially have set the date of operation to January 1, 1990, to avoid the confounding of calendar date (see Appendix I).]

ing is important because a risk factor always depletes the pool of high-risk patients as time evolves, thereby changing the prevalence of that factor in the remaining patient population. ${ }^{2}$ However, so long as a sufficient number of high-risk patients remain to permit risk factor identification, attrition by one event, such as death, does not unwittingly affect the analysis of another.

Reoperation and PTCA are outcomes after coronary bypass surgery whose occurrences are related to return of ischemic symptoms, decreased by attrition from death, and influenced by patients' decisions and physician recommendations when symptoms return. In this study, we have quantified only the influence of attrition by death on reintervention. We have not ascertained the contribution of patient-physician decision making. It is possible that this, rather than pathophysiology, may have contributed to the finding of a reduced incidence of reintervention in older patients. ${ }^{3,11}$

Is there a more insightful way to present event-free survival that is less counterintuitive? Yes.

In the setting of coronary artery disease, event-free survival gives rise to intellectually unappealing estimates of the same magnitude for a young patient, 
whose outcome is dominated by reintervention, and an older patient, whose outcome is dominated by death. The separate analysis of reoperation, PTCA, and death, followed by synthesizing them as competing risks, achieves the same overall result as event-free survival but, in addition, permits detailed examination and interpretation of each component's contribution to the overall outcome.

Competing risks. The answers to the specific questions posed for this study have required the use of a method of analysis and presentation that is uncommon in coronary bypass surgery, although occasionally employed in other cardiac surgical settings. ${ }^{9}, 10,13-15$ On occasion, single and multiple event analyses seemingly have been pitted against one another when, as in this study, they are complementary and based on the same underlying hazard functions. ${ }^{9,10}$

Competing risks analysis was described by Daniel Bernoulli 16 in 1760 to answer the question, "If in a given population smallpox could be eradicated, what would be the effect on the population mortality structure at different ages?" The analysis of time-related events was thereby generalized to consider not just isolated events, but the simultaneous consequences of multiple events. Bodnar, Haberman, and Wain ${ }^{17}$ introduced competing risks into the analysis of morbid events after heart valve replacement in 1979, calling it the name used in population demography, multiple decrement analysis. Grunkemeier and colleagues ${ }^{9,10}$ then introduced the designation "actual vs. actuarial," although we find this distinction troublesome.

\section{Limitations}

An important limitation of a competing risks analysis is that the mathematics treats each event, death, reoperation, or PTCA, as independent. That assumption may not be true. Death prevents subsequent reinterventions, but reinterventions may alter the subsequent risks of death. Since one can never know what reinterventions would have occurred had death been "eradicated," we have been able only to verify independence reasonably as regards death and death before reintervention (Appendix II). Methods for quantifying the influence of one event on another (so-called informative censoring) need further development and implementation. $^{18-20}$

We have limited our competing risks analysis to mutually exclusive end states. In fact, the technique has been generalized to an entire chain of events. ${ }^{15}$

Finally, calculations of actual benefits are related to length of follow-up. We have used 12 years as the time point for benefit assessment because the number of patients beyond that point was small. As patients age and follow-up increases, we anticipate more long-range benefits to accrue to those operated on at younger ages, and for whom death is not the major issue at the 12year follow-up interval.

We thank Penny Houghtaling, MS, for her biostatistical expertise, and Lucinda Mitchin for manuscript preparation. William N. Anderson, $\mathrm{PhD}$, wrote the SAS-based algorithms for the variance of the nonparametric competing risks estimates used in the figures, based on Andersen and associates. ${ }^{6}$

\section{REFERENCES}

1. Lytle BW, Blackstone EH, Loop FD, Houghtaling PL, Arnold JH, Akhrass R, et al. Two internal thoracic artery grafts are better than one. J Thorac Cardiovasc Surg 1999;117:855-72.

2. Sergeant P, Lesaffre E, Flameng W, Suy R, Blackstone E. The return of clinically evident ischemia after coronary artery bypass grafting. Eur J Cardiothorac Surg 1991;5:447-57.

3. Sergeant P, Blackstone E, Meyns B, Stockman B, Jashari R. First cardiological or cardiosurgical reintervention for ischemic heart disease after primary coronary artery bypass grafting. Eur $\mathrm{J}$ Cardiothorac Surg 1998;14:480-7.

4. David HA, Moeschberger ML. The theory of competing risks. New York: Macmillan; 1978. p. 45-56.

5. Kalbfleisch JD, Prentice RL. In: The statistical analysis of failure time data. New York: John Wiley; 1980. p. 163-88.

6. Andersen PK, Borgan O, Gill RD, Keiding N. In: Statistical models based on counting processes. Chap 4. Nonparametric estimation. Springer-Verlag; 1993. p. 176-331.

7. Kirklin JW, Barratt-Boyes BG. Cardiac surgery. Chap 6. Surgical concepts, research methods, and data analysis and use. New York: John Wiley; 1986. p. 177-204.

8. Blackstone EH, Naftel DC, Turner ME Jr. The decomposition of time-varying hazard into phases, each incorporating a separate stream of concomitant information. J Am Stat Assoc 1986;81:615-24

9. Grunkemeier GL, Jamieson WR, Miller DC, Starr A. Actuarial versus actual risk of porcine structural valve deterioration. J Thorac Cardiovasc Surg 1994;108:709-18.

10. Grunkemeier GL, Anderson RP, Miller DC, Starr A. Time-related analysis of nonfatal heart valve complications: cumulative incidence (actual) versus Kaplan-Meier (actuarial). Circulation 1997;96(Suppl):II-70-5.

11. Sergeant $P, B$ lackstone E, Meyns B. Is return of angina after coronary artery bypass grafting immutable, can it be delayed, and is it important? J Thorac Cardiovasc Surg 1998;116:440-53.

12. Sergeant P, Blackstone E, Meyns B. Validation and interdependence with patient-variables of the influence of procedural variables on early and late survival after CABG. K.U. Leuven Coronary Surgery Program. Eur J Cardiothorac Surg 1997;12:119.

13. McGiffin DC, Naftel DC, Kirklin JK, Morrow WR, Towbin J, Shaddy R, et al. Predicting outcome after listing for heart transplantation in children: comparison of Kaplan-Meier and parametric competing risk analysis. Pediatric Heart Transplant Study Group. J Heart Lung Transplant 1997;16:713-22.

14. Jacobs ML, Blackstone EH, Bailey LL, Congenital Heart Surgeons Society. Intermediate survival in neonates with aortic 
atresia: a multi-institutional study. J Thorac Cardiovasc Surg 1998;116:417-31.

15. Blackstone EH, Kirklin JW. Death and other time-related events after valve replacement. Circulation 1985;72:753-67.

16. Bernoulli D. Essai d'une nouvelle analyse de la mortalité causée par la petite Vérole, et des avantages de l'Inoculation pour la prévenir. Mém de L'Académie Royale de Science 1760:1-45.

17. Bodnar E, Haberman S, Wain WA. Comparative method for actuarial analysis of cardiac valve replacements. Br Heart J 1979;42:514-52.

18. Baker SG, Wax Y, Patterson BH. Regression analysis of grouped survival data: informative censoring and double sampling. Biometrics 1993;43:379-89.

19. Mori M, Woodworth GG, Woolson RW. Application of empirical Bayes inference to estimation of rate of change in the presence of informative right censoring. Stat Med 1992;11:621-31.

20. Wu MC, Carroll RJ. Estimation and comparison in the presence of informative right censoring by modeling the censoring process. Biometrics 1988;44:175-208.

\section{Discussion}

Dr Robert H. Jones (Durham, NC). Dr Blackstone has led our thinking in identifying the time hazard of risk and now leads the way in applying a 200-year-old technique, largely overlooked in medicine, that has promise for measuring the true benefit that our therapies provide. As our therapies become increasingly sophisticated, we trade off less pain or less cost, hoping to keep the survival the same, or we look at competing therapies that are just about equivalent on a few end points but may be different on others, such as PTCA and surgery. Proper comparison of expected outcomes with these techniques requires expression of the actual benefit that the patients can expect to receive in terms they can understand when they lie in the hospital bed and consider alternative therapies.

I predict that this concept applied to medicine will add as much benefit, if not more, than has our attention to the instantaneous hazard function that Dr Blackstone has showed us to be so valuable in his former work.

Life has been said to be a sexually transmitted, uniformly fatal condition. The implication of that to biostatisticians is that all survival curves converge on zero. The implication of that for cardiac surgeons is that we cannot be held completely responsible for preventing death. In fact, an ideal surgical operation would be one that deferred death from a cardiac reason until death could occur from another reason, particularly if no further cardiac interventions were required after the initial operation.

Unless we were very sophisticated biostatisticians, last year we went home from this meeting and, on the basis of these same data, said to our patients with coronary disease that they had dramatic survival benefit and lower reintervention benefit from coronary bypass in which two ITAs were used compared with one. To have been completely accurate, we would have said, "If you live for 12 years, your reintervention procedure rate will be $8 \%$ with bypass surgery and $7 \%$ with PTCA." The patient might have asked us, "But, doctor, what are my chances of dying over this 12-year period that you are talking about?"
And you would have said, "27\%, but one third of those deaths are not things I can prevent because they are noncardiac." This would have been a very confusing but accurate conveyance of this same information. This year you can go home and tell your patients, "If I do two ITA grafts instead of one, over the next 12 years of your life you have $2 \%$ less chance of death, $2 \%$ less chance of reintervention with bypass surgery, and $2 \%$ less chance of revascularization again with PTCA. That's six big things out of a hundred that you would rather not have happen to you over the next 12 years that summarizes the total benefit to you if I do a second ITA graft."

I do not know what this says to you, but it says to me that my standard operation has changed from this day forward: from a single ITA graft, because of lack of real valid evidence as to the magnitude of benefit, to a standard of two ITA grafts, because I can quantitate the benefit in a way patients understand that is accurate and applicable. This is a dramatic contribution to our profession. I congratulate the authors.

Dr Blackstone. Thank you very much, Dr Jones, for those kind remarks. Coming from a person like you that I respect very much, this means more than you can imagine.

Allow me one further refinement of your comments. Not all patients will receive $2+2+2$ benefits from the use of both ITAs. Some will receive less and others more. Unfortunately, identifying who will benefit more and who will benefit less is not simple to generalize, even though we have emphasized age as one of those considerations in this presentation. However, the equations presented are actually perfectly capable of being used for quantifying the benefit for an individual patient.

\section{Appendix I: Confounding by changing mode of reintervention}

During the time frame of this study, the modes of reintervention evolved from reoperation only to either reoperation or percutaneous interventions (Appendix Fig 1). The correlates of PTCA being the mode of reintervention included more recent date of operation, bilateral versus single ITA grafting, left anterior descending coronary artery disease for which ITAs were placed to this artery, and younger age at operation (Appendix Table I). In contrast, a reoperation was more likely to be the reintervention earlier in the experience, in older patients, in those with only a single ITA and vein grafts, and in left main disease.

\section{Appendix II: Transformation of probability estimates to competing risks estimates}

Using compartmental theory, which can be generalized from unidirectional biochemical reactions to competing risks, we assumed that (1) death before reintervention, reintervention by reoperation, and reintervention by PTCA were independent, (2) patients are conserved in competing risks analyses just as mass is conserved in biochemical reactions, and (3) all patients start in the category "alive without reintervention." Because the parametric modeling method yields an equation for continuous time, the differential equations gov- 


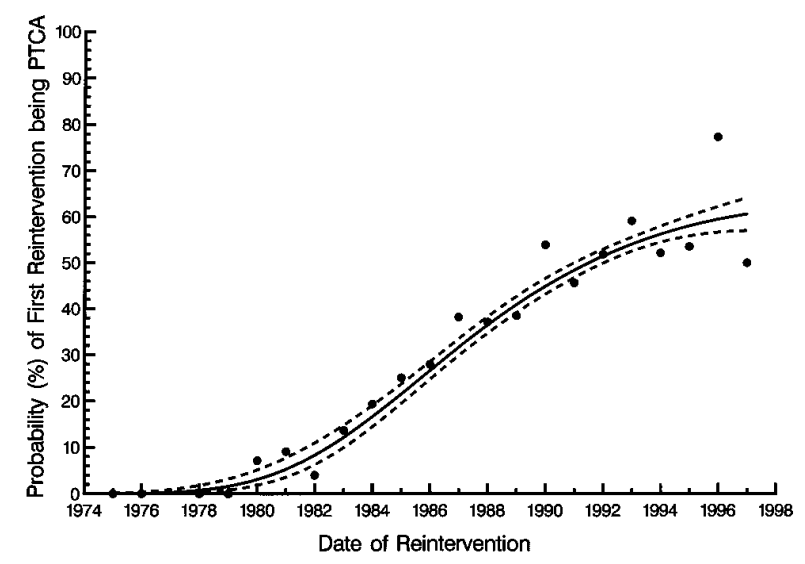

Appendix Fig 1. Probability of first reintervention being a PTCA according to the date of reintervention. The filled circles are the actual proportions of patients receiving a PTCA in each calendar year. The solid line and its asymmetric $68 \%$ confidence limits (equivalent to one standard error) are from a univariable logistic regression analysis of these data.

erning the process were set up and solved as for any compartmental model.

Thus, for the category (compartment) death $\mathrm{C}_{\mathrm{D}}(\mathrm{t})$, reoperation $\mathrm{C}_{\mathrm{R}}(\mathrm{t})$, and $P T C A \mathrm{C}_{\mathrm{P}}(\mathrm{t})$, the change (increase) in number of patients with time $t$ will be related to the respective transition rates (hazard functions) $\lambda_{D}(t), \lambda_{R}(t)$, and $\lambda_{P}(t)$ as they draw patients from the category alive without reintervention $\mathrm{C}_{\mathrm{A}}(\mathrm{t})$ :

$$
\begin{gathered}
\frac{\delta C_{D}(t)}{\delta t}=\lambda_{D}(t) C_{A}(t) \\
\frac{\delta C_{R}(t)}{\delta t}=\lambda_{R}(t) C_{A}(t) \\
\frac{\delta C_{p}(t)}{\delta t}=\lambda_{p}(t) C_{A}(t)
\end{gathered}
$$

Conservation of patients means that if the equations above are normalized to 100 patients, at any time t:

$$
C_{A}(t)+C_{D}(t)+C_{R}(t)+C_{P}(t)=100
$$

Independence also implies that the hazard function for event-free survival will be:

$$
\lambda_{A}(t)=-\lambda_{D}(t)-\lambda_{R}(t)-\lambda_{P}(t)
$$

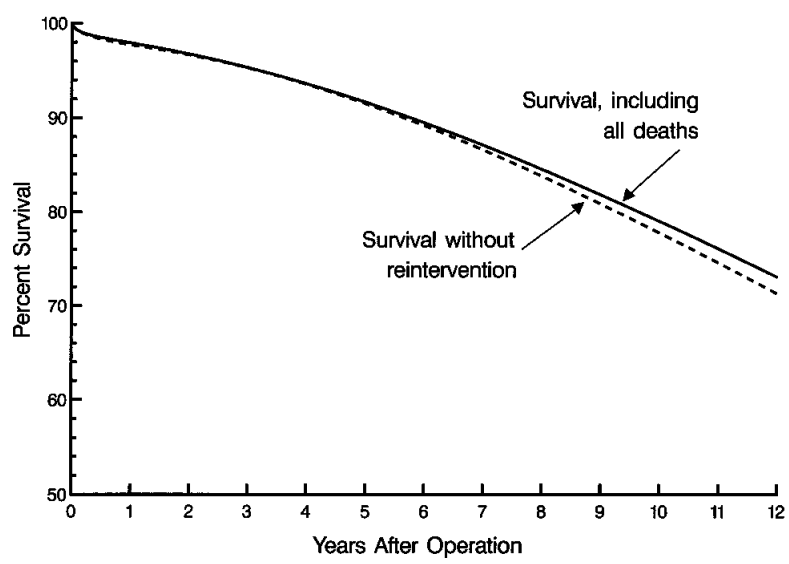

Appendix Fig 2. Comparison of survival without reintervention (competing risks analysis) to survival that includes all deaths. The close correspondence partially validates the assumption of independence underlying the competing risks calculations used in this study.

Finally, CA(t) can be thought of as surviving $\left[\mathrm{S}_{\mathrm{D}}(\mathrm{t})\right]$, being free of reoperation $\left[\mathrm{S}_{\mathrm{R}}(\mathrm{t})\right]$, and being free of PTCA $\left[\mathrm{S}_{\mathrm{P}}(\mathrm{t})\right]$ :

$$
C_{A}(t)=S_{D}(t) S_{R}(t) S_{P}(t)
$$

where:

$$
S_{i}(t)=e^{-\int{ }_{0}^{t} \lambda_{i}(u) d u}
$$

The survivorship functions were obtained analytically by the parametric survival analysis system.

With the use of all this information, integration to obtain $C_{D}(t), C_{R}(t)$, and $C_{P}(t)$ was performed numerically.

\section{Appendix III: Investigation of possible informative censoring}

An assumption of this competing risks analysis was that each of the events, and therefore their corresponding hazard functions, was independent one from the other. This has sometimes been called noninformative censoring, because migration from the category alive without reintervention into any event category behaves as a censoring mechanism for all other event categories. One test of the reasonableness of this assumption of independence is the comparison of survival without reintervention to overall survival, the latter including all deaths at all times, including those subsequent to reinterventions (Appendix Fig 2). 
Appendix Table I. Correlates of the use of PTCA rather than reoperation at the first reintervention after CABG

\begin{tabular}{|c|c|c|c|}
\hline Correlates of PTCA as first intervention & Logistic coefficient $\pm S D$ & Direction of influence & $\mathrm{P}$ \\
\hline \multicolumn{4}{|l|}{ Demographic } \\
\hline Age at initial CABG in bilateral ITA group* & $-1.55 \pm 0.66$ & $\downarrow$ & .02 \\
\hline \multicolumn{4}{|l|}{ Coronary disease } \\
\hline Left main disease ( $\geq 50 \%$ diameter obstruction) $)^{\dagger}$ & $-0.64 \pm 0.193$ & $\downarrow$ & .0009 \\
\hline LAD system disease ( $\geq 70 \%$ diameter obstruction) & $0.41 \pm 0.169$ & $\uparrow$ & .01 \\
\hline RCA system disease $(\geq 70 \% \text { diameter obstruction })^{\dagger}$ & $-0.48 \pm 0.123$ & $\downarrow$ & $<.0001$ \\
\hline LCx system disease $(\geq 70 \% \text { diameter obstruction })^{\dagger}$ & $-0.31 \pm 0.116$ & $\downarrow$ & .008 \\
\hline \multicolumn{4}{|l|}{ Operation } \\
\hline Bilateral ITA grafting & $2.6 \pm 0.90$ & $\uparrow$ & .003 \\
\hline ITA to LAD & $0.56 \pm 0.194$ & $\uparrow$ & .004 \\
\hline ITA to $\mathrm{LCX}$ & $0.77 \pm 0.30$ & $\uparrow$ & .01 \\
\hline Date of operation & $0.69 \pm 0.22$ & $\uparrow$ & .001 \\
\hline Date of reintervention & $4.1 \pm 0.76$ & $\uparrow$ & $<.0001$ \\
\hline And date of operation ${ }^{\S}$ & $-0.20 \pm 0.073$ & $\downarrow$ & .006 \\
\hline
\end{tabular}

$P T C A$, Percutaneous transluminal coronary angioplasty; $S D$, standard deviation; $C A B G$, coronary artery bypass grafting; ITA, internal thoracic artery; $L A D$, left anterior descending coronary artery; $R C A$, right coronary artery; $L C x$, left circumflex coronary artery.

* $[\text { age } / 50]^{2}$ transformation.

'Negative sign of coefficient indicates that the presence of this factor is associated with a lower chance of PTCA versus reoperation.

$\div \ln$ [number of years to reintervention since January 1971] logarithmic transformation.

$\S$ Interaction of date of operation and logarithmic transformation of date of reintervention.

\section{Targeted}

The Journal of Thoracic and Cardiovascular Surgery gives you two tables of contents.

The condensed table of contents tells you at a glance what topics and authors are presented each month. The expanded table of contents gives you a brief abstract of each article. You select only those articles of most interest to you for further reading. 